\title{
Radiated and Conducted EMI by RF Fields at Hospital Environment
}

\author{
Jamilson Evangelista \\ Department of Electronics and \\ Biomedical Engineering \\ University of Campinas \\ Campinas, Brazil \\ jamilson.ramos@gmail.com \\ Robert Smolenski \\ Institute of Automatic Control, \\ Electronics and Electrical Engineering \\ University of Zielona Góra \\ Zielona Góra, Poland \\ r.smolenski@iee.uz.zgora.pl
}

\author{
Hermes Loschi* \\ University of Zielona Góra \\ University of Twente \\ University of Nottingham \\ Zielona Góra, Poland \\ eng.hermes.loschi@ieee.org
}

\author{
Niek Moonen \\ Faculty of Electrical Engineering, \\ Mathematics and Computer Science \\ University of Twente \\ Enschede, Netherlands \\ niek.moonen@utwente.nl
}

\author{
Eduardo Tavares Costa \\ Department of Electronics and \\ Biomedical Engineering \\ University of Campinas \\ Campinas, Brazil \\ educosta@unicamp.br
}

\author{
Robert Vogt-Ardatjew \\ Faculty of Electrical Engineering, \\ Mathematics and Computer Science \\ University of Twente \\ Enschede, Netherlands \\ r.a.vogtardatjew@utwente.nl
}

\begin{abstract}
Nowadays, hospital environments are among the most complex environments to establish electromagnetic compatibility. The newest health technologies associated with medical electrical equipment and their possible electromagnetic interference are being extensively investigated. However, the electricity supply system infrastructure remains present in hospital environments and might be responsible for the conducted electromagnetic interference through coupling with radio frequency fields, e.g. broadcasting station. Therefore, aiming to understand the electromagnetic environment, this paper presents the methodology used and the electromagnetic interference measurements in one hospital environment in São Paulo, Brazil. The results show that although all equipment in surgery room has been tested for electromagnetic compatibility, the risk still exists. Highlighting how important the comprehension and analysis of the electromagnetic environment is as a first step towards implementing specific solutions to mitigate the medium and high risk cases.
\end{abstract}

Index Terms-electromagnetic compatibility, radiated and conducted electromagnetic interference, hospital, risk-based EMC, clinical engineering.

\section{INTRODUCTION}

$\mathbf{S}$ INCE the mid-1990s the risk of electromagnetic interference (EMI) due to the increasing number of medical electrical equipment (MEE) in the professional healthcare facility environment has been continuously reported [1]. Despite manufacturers' efforts to enhance the MEE conformity, new challenges are introduced to the electromagnetic compatibility (EMC) based on the newest health technologies [2], [3]. The trend is to define the MEE regulations directly at manufacturers to ensure conformity and EMC. On the other hand, the risk

This paper has received funding from the European Union's Horizon 2020 research and innovation programme under the Marie Skłodowska-Curie grant agreement No.812391 - SCENT. :

*Corresponding author: eng.hermes.loschi@ieee.org (Hermes Loschi). management processes [4] in professional healthcare facility environments ensure patient safety when using the MEE.

According to the IEC 60601-1-2 standard [5], the hospital is considered a professional healthcare facility location and must have a controlled electromagnetic (EM) environment concerning fixed radio frequency (RF) electromagnetic sources. Thus, the Brazilian National Health Surveillance Agency (Agência Nacional de Vigilância Sanitária - ANVISA) established hospital obligation to implement a system for monitoring and managing risk-based EMC to their health technologies. This system aims to reduce and minimize incidents without damage (event or circumstance that could have resulted in unnecessary damage to patient or operator health), adverse events (incidents that resulted in damage to patient or operator health), and near error or near miss (incidents that occurred, but did not reach the patient or equipment operator) [6]. However, the approach of risk-based EMC to the health technologies must also cover the atypical situation of radiated EMI from RF fields, e.g. broadcasting station, which might induce the conducted EMI [7], through the coupling with the electricity supply system infrastructure.

The IEC 60364-7-710 standard [8] applies to electrical installations in hospital environments to ensure safety of patients and medical staff, establishing requirements for components, such as uninterruptible power supplies (UPS), transformer, filtering, as well as the whole electrical installation [9]. Nevertheless, some hospital environments consider "machine or equipment room" outside the main building or with a low attenuation infrastructure, which might favor the coupling with radiated EMI by outside RF fields. Furthermore, if the electrical installation uses long-distance cables without EMI filters, may favor the conducted EMI flow.

Therefore, aiming to understand the EM environment, this 
paper presents a methodology and its application, performing EMI measurements in a hospital environment in São Paulo city, Brazil. The paper is organized as follows: in Section II, the hospital environment and electricity supply system are described. Then in Section III, the radiated and conducted EMI measurements are presented. Section IV we make some considerations based on the risk-based EMC assessment, and in Section V the paper conclusion.

\section{HOSPITAL ENVIRONMENT}

In a certain region of São Paulo city, there are 24 broadcasting stations (FM radio and TV) with radiated power from $20 \mathrm{~kW}$ to $360 \mathrm{~kW}$ [10]. More than half of these stations are located in $1.5 \mathrm{~km}^{2}$ commercial area, including at least 10 professional healthcare facilities. This area is known as the Av. Paulista. Table I shows all the broadcasting systems (FM and TV stations) located near $\left(<1.5 \mathrm{~km}^{2}\right)$ Av. Paulista.

TABLE I

THE BROADCASTING SYSTEMS LOCATED NEAR $\left(<1,5 \mathrm{~km}^{2}\right)$ OF AV. PAUlista [10].

\begin{tabular}{|c|c|c|c|}
\hline FM Station & Frequency & TV & UHF Digital \\
\hline GAZETA & $88.1 \mathrm{MHz}$ & CBI & $482-488 \mathrm{MHz}$ \\
\hline RADIO ROCK & $89.1 \mathrm{MHz}$ & GAZETA & $488-494 \mathrm{MHz}$ \\
\hline NOVA BRASIL & $89.7 \mathrm{MHz}$ & GLOBO & $494-500 \mathrm{MHz}$ \\
\hline CBN & $90.5 \mathrm{MHz}$ & RECORD & $506-512 \mathrm{MHz}$ \\
\hline ANTENA 1 & $94.7 \mathrm{MHz}$ & REDE 21 & $518-524 \mathrm{MHz}$ \\
\hline REDE ALELUIA & $99.5 \mathrm{MHz}$ & BANDEIRANTES & $524-530 \mathrm{MHz}$ \\
\hline KISS & $102.1 \mathrm{MHz}$ & REDE GOSPEL & $548-554 \mathrm{MHz}$ \\
\hline TUPI & $104.1 \mathrm{MHz}$ & -- & -- \\
\hline MIX & $106.3 \mathrm{MHz}$ & -- & -- \\
\hline
\end{tabular}

We have selected two hospital environments, case study of this paper, considering their location near to the broadcasting stations described in Table I. We have investigated how the radiated EMI from the RF fields may induce the conducted EMI through the coupling with the electricity supply system infrastructure. Two surgery rooms in the hospital were selected:

- Surgery room 1: In this surgery room there are the MEE to be evaluated, such as computer, anesthesia system, multiparametric monitor, and endoscope. This surgery room was also chosen due to fact that it is distant, hence connected with long cables to the electrical panel (the transformer). This is important because the transformer may act as a low-pass filter (LPF) for the conducted EMI. Moreover, this surgery room is located on the north side of the hospital building, which means long distance from Av. Paulista.

- Surgery room 2: This surgery room is the closest to the machine room. The machine room contains a UPS $15 \mathrm{kVA}-13.2 \mathrm{~kW}$, with sealed lead-acid batteries and calculated autonomy of 15 minutes at a load of $13.2 \mathrm{~kW}$. Also, this surgery room is located on the south side of the hospital building, which means a small distance from to Av. Paulista.

Therefore, the radiated EMI measurement was first carried out in Surgery room 2, next to the machine room, to quantify the RF field magnitude. Secondly, the conducted EMI measurement was carried out on the electrical system to identify the contribution of each MEE present in Surgery room 1. All the radiated and conducted EMI measurements were performed in Surgery room 1 and Surgery room 2 without medical procedures.

\section{RADIATED AND CONDUCTED EMI MEASUREMENTS}

Based on the IEC 60601-1-2 standard [5], the RF radiated electromagnetic immunity limits to the hospital environment is $130 \mathrm{~dB} \mu \mathrm{V}$. Therefore, this limit of $130 \mathrm{~dB} \mu \mathrm{V}$ was considered as a reference value for the radiated EMI measurement carried out from $30 \mathrm{MHz}$ to $1 \mathrm{GHz}$ with the intermediate frequency bandwidth (IFBW) equal to $200 \mathrm{kHz}$. Fig. 1 shows the setup of the radiated EMI measurement in Surgery room 2, next to the machine room.

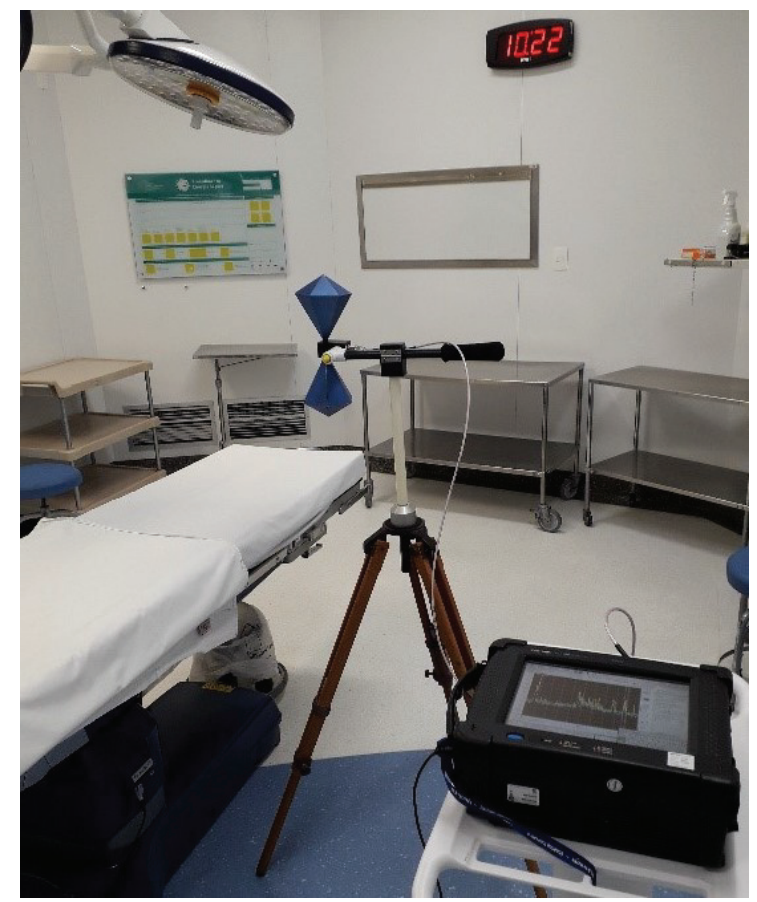

Fig. 1. The setup of the radiated EMI measurement in Surgery room 2, next to the machine room.

The radiated and conducted EMI measurements were carried out with a Tektronix SA2600 spectrum analyzer. However, a current probe Fisher FCC-33-1 was used for the conducted EMI, and a biconical radial isotropic antenna (BicoLog 203000) was used for the radiated EMI measurement (Fig. 1). In addition, the EM field levels were recorded in four positions $\left(0^{\circ}, 90^{\circ}, 180^{\circ}\right.$, and $\left.270^{\circ}\right)$ and two polarization (vertical and horizontal) around the antenna stand.

Fig. 2 shows the conducted EMI measurement point on the input of the multiparametric monitor (MM) in Surgery room 1. It is worthy to note that the Surgery room 1 contains also the endoscope (EN), anesthesia system (AS), and computer (CP). Nevertheless, to evaluate the electricity supply system, the conducted EMI measurements were taken 
in the alternating current supply of the Surgery room 1 for each MEE. The conducted EMI measurements were carried out from $150 \mathrm{kHz}$ to $150 \mathrm{MHz}$ with the IFBW equal to $9 \mathrm{kHz}$.

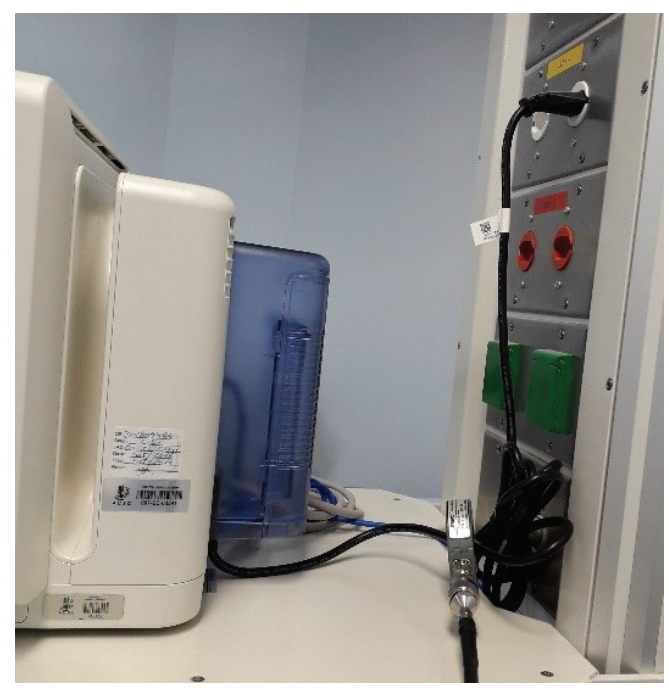

Fig. 2. Conducted EMI measurement point on the input electrical system of Surgery room 1.

Fig. 3 shows the radiated EMI measurement in Surgery room 2, next to the machine room, for the four positions $\left(0^{\circ}, 90^{\circ}, 180^{\circ}\right.$, and $\left.270^{\circ}\right)$ and vertical $(\mathrm{V})$ and horizontal $(\mathrm{H})$ polarization around the antenna stand.

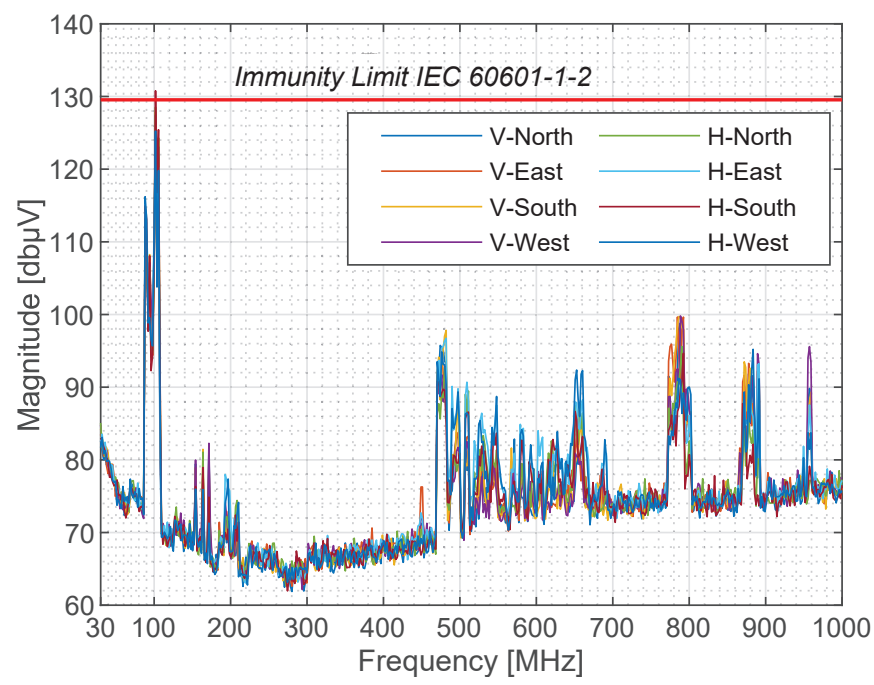

Fig. 3. Radiated EMI measurement in Surgery room 2, next to the machine room - IFBW=200kHz.

The RF fields related to FM radio, mentioned in Table I, were identified by the measurements presented in Fig. 3, around $100 \mathrm{MHz}$. Also, RF fields related to ultra-high frequency (UHF) Digital TV (Table I) were also identified by the measurements presented in Fig. 3. It is worthy to note that the radiated EMI measurements values presented in Fig. 3 were corrected considering the correction factors of the antenna BicoLog 203000.

Fig. 4 shows the conducted EMI measurement in Surgery room 1 with the contribution of each MEE.

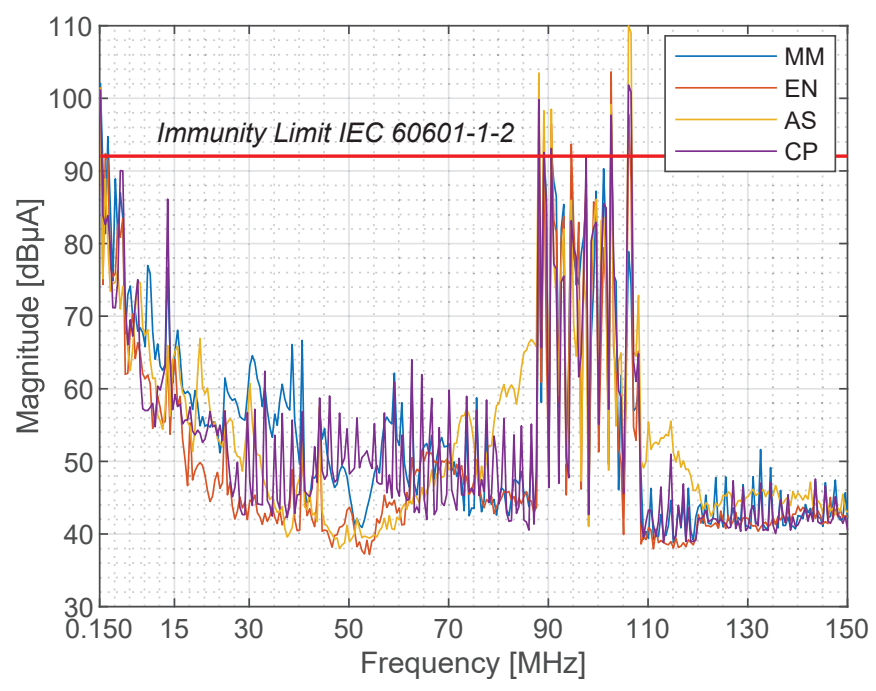

Fig. 4. Conducted EMI measurement in Surgery room 1 with the contribution of each MEE: multiparametric monitor (MM), endoscope (EN), anesthesia system (AS), and computer (CP).

The measurements shown in Fig. 4 considers the contribution of all the equipment of Surgery room 1. In all cases, it is possible to observe the conducted EMI induced by the coupling phenomenon, especially because all the high levels of $\mathrm{dB} \mu \mathrm{A}$ are related to $\mathrm{FM}$ stations, mentioned in Table I, around $100 \mathrm{MHz}$.

Furthermore, the measurement values (Fig. 4) were corrected, considering the correction factors of the current probe Fisher FCC-33-1. It is also noteworthy that the voltage limit of $6 \mathrm{Vrms}$, based on the IEC 60601-1-2 standard [5] and also presented in Fig. 4, was considered as a reference value for the conducted EMI measurement. Therefore, the quantities were converted to become $92 \mathrm{~dB} \mu \mathrm{A}$. The use of the current probe Fisher FCC-33-1, did not allow us to determine the RF impedance of the equipment power input.

\section{Discussion}

The risk-based EMC assessment might assume that a MEE can significantly influence an adverse event. For example, a MEE may appear to function properly after an adverse event. However, still, it may have been the cause of a functional failure in the hospital environment. Therefore, the risk-based EMC might be defined from the lowest MEE immunity level and the highest identified disturbance level (Table II) [11].

Table III presents quantitative data with the worst situation based on the EMI measurements, presented in Fig. 3 and Fig. 4.

Based on Table III and considering the IEC 60601-1-2 standard [5], which defines the RF radiated electromagnetic immunity limits for MEE used in hospital environment equal 
TABLE II

RISK CLASSIFICATIONS.

\begin{tabular}{|c|c|}
\hline Risk & Classifications \\
\hline Low & Disturbance $<$ Immunity \\
\hline Medium & Disturbance $=$ Immunity \\
\hline High & Disturbance $>$ Immunity \\
\hline
\end{tabular}

TABLE III

COMPILATION OF DATA WITH THE WORST REASONABLY FORESEEABLE SITUATION

\begin{tabular}{|c|c|c|c|}
\hline \multicolumn{2}{|c|}{ Disturbance } & \multicolumn{2}{c|}{ Immunity } \\
\hline Type & Level & Origin & Level \\
\hline Radiated EMI & $130 \mathrm{~dB} \mu \mathrm{V}$ & Measurement & $130 \mathrm{~dB} \mu \mathrm{V}$ \\
\hline Conducted EMI & $110 \mathrm{~dB} \mu \mathrm{A}$ & Measurement & $92 \mathrm{~dB} \mu \mathrm{A}$ \\
\hline
\end{tabular}

to $130 \mathrm{~dB} \mu \mathrm{V}$ for radiated and $92 \mathrm{~dB} \mu \mathrm{A}$ for conducted EMI, it can be seen that the functional risk might be considered medium for radiated EMI and high for the conducted EMI.

On the other hand, the phenomenon of radiated EMI by outside RF fields, based on Table III, induced the conducted EMI through the coupling with the electricity supply system infrastructure. Basically, due to the transfer impedance, admittance parameters and the imminence of propagation by the cable's internal wiring, the induced voltages and currents (depending on loads) between wire pairs (coupling in differential mode) can occur. This is especially true in electrical installations with long-distance cables, without EMI filters or transformers near to surgery room electrical installation system, which may act as an LPF.

\section{CONCLUSion}

The risk management processes in professional healthcare facility environments ensure patient safety when using the MEE suited for the electromagnetic environment. Thus, this paper presents an atypical situation with radiated EMI by outside RF fields, i.e., broadcasting systems, which can raise an EMC-based risk condition. It stands out that although all equipment in Surgery room 1 has been tested for EMC, the risk still exists, which confirms how important is comprehension and analyze the EM environment.

Through a quick and simple EMI measurement, the phenomenon of inducing conducted EMI through coupling with the electricity supply system infrastructure was highlighted. Our case study classified the risk as medium for radiated EMI and high for the conducted EMI. However, it is noteworthy that the precise definition of EMC-based risk must consider implementing specific solutions to mitigate medium and high risk cases, such as performing spatial mapping and using longer measurement times. Especially because the EM fields could be even higher or smaller due to reflections or when measured at a different time.

Prospectively, in order to mitigate the medium risk for radiated EMI and high risk for the conducted EMI, some general recommendations can be adopted:
- Shielding to attenuate the level of radiated electromagnetic disturbances in the FM and TV band inside the surgery rooms;

- Filtration in the electric power supply to attenuate the level of conducted electromagnetic disturbances in the range of $150 \mathrm{kHz}$ to $250 \mathrm{MHz}$ in the surgery rooms.

Additionally, clinical engineering professionals with proper and increasing understanding and knowledge on riskmanagement EMI is needed to cope with the ever increasing challenges in this field.

\section{REFERENCES}

[1] J. L. Silberberg, "An FDA Perspective on Medical Device EMC and Wireless WED-PM-4," 2018 IEEE Symposium on Electromagnetic Compatibility, Signal Integrity and Power Integrity (EMC, SI and PI), Long Beach, CA, 2018, pp. 1-84, doi: 10.1109/EMCSI.2018.8495328.

[2] M. Das, S. Jeunink, R. Vogt-Ardatjew, B. van den Berg and F. Leferink, "Introduction of Wireless Services and Devices in a Hospital Environment Following a Risk-based EMC Approach,” 2020 International Symposium on Electromagnetic Compatibility - EMC EUROPE, Rome, Italy, 2020, pp. 1-6, doi: 10.1109/EMCEUROPE48519.2020.9245878.

[3] M. Das, R. Vogt-Ardatjew, B. van den Berg and F. Leferink, "Risk-based EMC Approach in Hospital Environment," 2020 IEEE International Symposium on Electromagnetic Compatibility and Signal/Power Integrity (EMCSI), Reno, NV, USA, 2020, pp. 676-680, doi: 10.1109/EMCSI38923.2020.9191637.

[4] F. Leferink, "Risk-based vs Rule-based Electromagnetic Compatibility in Large Installations," 2018 IEEE 4th Global Electromagnetic Compatibility Conference (GEMCCON), Stellenbosch, South Africa, 2018, pp. 1-4, doi: 10.1109/GEMCCON.2018.8628505.

[5] IEC, 60601-1-2:2014, "Medical electrical equipment - Part 1-2: General requirements for basic safety and essential performance - Collateral Standard: Electromagnetic disturbances - Requirements and tests".

[6] Brazil. Ministry of Health. National Health Surveillance Agency. Technovigilance Manual: approaches to health surveillance of health products marketed in Brazil. - Brasília: Ministry of Health, 2010. 629 p

[7] F. Leferink, "Conducted Interference, Challenges and Interference Cases," in IEEE Electromagnetic Compatibility Magazine, vol. 4, no. 1, pp. 78-85, 1st Quarter 2015, doi: 10.1109/MEMC.2015.7098517.

[8] IEC, 60364-7-7-710:2006, "Electrical installations of buildings - Part 7-710: Requirements for special installations or locations - Medical locations"

[9] M. Stroili, E. C. Pavan, M. Gorela and F. Kenda, "The dimensioning and development of hospital electric installations to guarantee the continuity of use of the therapeutic and diagnostic system," 2015 37th Annual International Conference of the IEEE Engineering in Medicine and Biology Society (EMBC), Milan, 2015, pp. 1211-1214, doi: 10.1109/EMBC.2015.7318584.

[10] SIGAnatel - Sistema de Informações Geográficas. ANATEL. Brasilia, DF, Brasil. March 9,2016 [Online] Available: https://sistemas.anatel.gov.br/siganatel

[11] IEC TR 61000-2-5:2017, "Electromagnetic compatibility (EMC) - Part 2-5: Environment - Description and classification of electromagnetic environments" 